\title{
Regulation of Oxidative Imbalances in Mitochondria and Endoplasmic Reticulum
}

\author{
Kishu Ranjan ${ }^{1 *}$ and Saroj Kumar Amar ${ }^{2,3 *}$ \\ ${ }^{1}$ Department of Internal Medicine, Yale University, USA \\ ${ }^{2}$ School of Medicine, Yale University, USA \\ ${ }^{3}$ School of Bioengineering and Biosciences, Lovely Professional University, India
}

*Corresponding author: Kishu Ranjan, Department of Internal Medicine, Section of Digestive Disease, Yale University, New Haven, USA.

Saroj Kumar Amar, School of Medicine, Yale University, New Haven, USA, School of Bioengineering and Biosciences, Lovely Professional University, Punjab, India.

To Cite This Article: Kishu Ranjan, Saroj Kumar Amar, Regulation of Oxidative Imbalances in Mitochondria and Endoplasmic Reticulum. Am J Biomed Sci \& Res. 2020 - 7(2). AJBSR.MS.ID.001124. DOI: 10.34297/AJBSR.2020.07.001124.

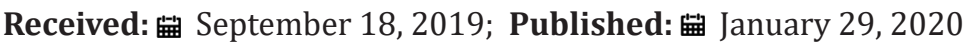

\begin{abstract}
Communications between cell organelles are pivotal to resolve cellular stress and dysregulated signaling. The physiological processes inside the cells generates several byproducts that may impose stress to the cells. Indeed, mitochondrial dysfunction during biochemistry of ATP synthesis generates excessive reactive oxygen species (ROS) that may cause cellular aging and disease. The mitochondrial electron transport chain (ETC) associated respiratory complexes are the major source of reactive oxygen species (ROS) and imbalance in ROS production results in stress to the cells. The mitochondrial anti-oxidant defense system alleviates the oxidative burden through converting reactive radicals into stable molecules. On the other hand, endoplasmic reticulum (ER) serves as a prime site for protein synthesis also generates oxidative stress due to immature protein folding. The ER lumen anchored protein such as protein disulfide isomerase (PDI) enzymes catabolize the disulfide bond formation in the newly synthesize proteins, but any perturbation in this process leads to oxidative imbalance in the cells. Notably, the interface of mitochondrial and ER adds a new dimension in the feedback regulation of oxidative stress. This review highlights the role of mitochondria and ER during oxidative stress and the feedback regulation of restoring $\mathrm{Ca}^{2+}$ balance.
\end{abstract}

Keywords: Oxidative stress, Electron transport chain, Calcium signaling, UPR

Abbreviations: ETC: Electron Transport Chain; ERAD: ER-Associated Degradation; ERO ${ }_{-1}$ : Endoplasmic Reticulum Oxidoreductin; FMN: Flavin Mononucleotide Group; MMP: Mitochondrial Membrane Potential; IP3: Inositol 1,4,5-trisphosphate; mNCX: $\mathrm{Na}^{+} / \mathrm{Ca}^{2+}$ Exchanger; SERCA: SarcoEndoplasmic Reticulum $\mathrm{Ca}^{2+}$-ATPase; RyR: Ryanodine Receptor; $\mathrm{XBP}_{1}$ : X-box Binding Protein 1

\section{Introduction}

Intracellular oxidative stress has been described as a double-edged sword, at the physiological level these molecules complements cellular functioning, but excessive burden may cause damaged to cellular macromolecules and organelles [1,2]. Notably, cells dissipate the reactive oxygen species (ROS) induced oxidative stress through arsenal of antioxidant enzymes (such as glutathione peroxidase, catalase, thioredoxin reductase and superoxide dismutase) and by redox systems (such as oxidized glutathione/ reduced glutathione (GSSG/GSH), NAD+/NADH, NADP+/NADPH) to maintain cellular homeostasis [3]. Interestingly, the oxidative imbalance in the cells are mostly generated through organelles in the process of building cellular architecture [4,5]. For instance, the mitochondrial respiratory chain or electron transport chain (ETC) comprises of complex I to IV are the prime site for production of reactive oxygen species (ROS). The mitochondrial ETC generates superoxide anion that converts to hydrogen peroxide $\left(\mathrm{H}_{2} \mathrm{O}_{2}\right)$ through mitochondrial dismutase and may generates highly reactive hydroxyl radical such as super oxide $\left(\mathrm{O}_{2} \bullet-\right)$ and hydroxyl radical $(\mathrm{OH} \bullet)[5,6]$. Similarly, reactive nitrogen species (RNS) is derived from nitrogen species also known as peroxynitrite (ONOO-) in the term of ions. 
Thus, nitric oxide (NO·) which is also derives from mitochondria (NO•), can alter the cellular activity like respiration, oxidative stress and mitochondrial biogenesis by enhancing the production of ROS and RNS [7]. Therefore, the term oxidative stress represents both ROS and RNS. On the other side, organelles such as peroxisome and the endoplasmic reticulum (ER) at the physiological levels generates oxidative stress within in the cells [8]. The exacerbated accumulation of terminally misfolded proteins been removed through activation of unfolded protein response (UPR) and ERassociated degradation (ERAD) pathway to restore homeostasis [9]. In fact, immature protein folding within the oxidized lumen of ER generates oxidative stress within the cells [10]. Importantly, the enzymatic cascade of endoplasmic reticulum oxidoreductin $\left(\mathrm{ERO}_{-1}\right)$, and NADPH oxidase complexes balances redox levels of ER lumen to regulate oxidative stress in the cells [11]. However, failure to resolve mitochondrial and ER induced oxidative burden leads to cell death $[12,13]$.

\section{Mitochondrial ETC complex: source of reactive radicles}

The role of mitochondrial complex I and III are more significant in the production of ROS [14]. Although the major site for ROS production under oxidizing complex I is still not clear [15]. Previous studies showed that pathological and physiological site site in complex I for ROS is flavin mononucleotide group (FMN) not the ubiquinone of complex III $[16,17]$. For the production of superoxide radicals $(02 \bullet-)$, Complex III might be more responsible specially from the mitochondria of heart and lungs [18], but the superoxide formation with complex I might be the primary source for mitochondria of brains [19]. The production of ROS is measured in term of $\mathrm{H}_{2} \mathrm{O}_{2}$ because of quenching property of mitochondrial superoxide dismutase (SOD), which quickly control the superoxide production and finally dismutated by SOD. Thus, the production of superoxide/ROS has been measured in term of $\mathrm{H}_{2} \mathrm{O}_{2}$ only. Although the enzymatic source of super oxide is NADPH oxidase which can be present on endothelial cells, microphases and cell membrane [20]. To confirm the production site in different complexes of respiratory chain several study have been reported, study reveals that rotenone quencher of complex I not enhancing the ROS generation in mitochondria but very significantly increased in submitochondrial particles linked with NADH oxidation [21]. In the pathogenesis of myocardial ischemia, negative effect of rotenone in the production of ROS have been reported [22]. Study further reveals that when complex III is ceased by antimycin A, then the oxidation of both complex I and II favor the generation of ROS [23]. Proper balance between ROS generation and anti-oxidant defenses is required to control the pathogenesis linked with various disease. The balance concentration of anti-oxidant in mitochondria is mentioned by anti-oxidant defense and repair enzyme [18]. Mitochondrial membrane potential (MMP) is another evidence of ROS induced damage of inner membrane of mitochondria. Lower the MMP higher the chance of impairment of inner membrane of mitochondria, which favor the release of apoptotic signals like Bax and cell death via final activation of Caspase $_{3}$ [24]. Our previous report also concluded that release of $\mathrm{Ca}^{++}$from endoplasmic reticulum (ER) upregulated the stress marker of ER like AFT6 and CHOP at gene level. CHOP directly correlate the MMP and finally active the chain of caspase via elevated level of $\mathrm{Bax} / \mathrm{Bcl}_{2}$ ratio [25]. Effect of xenobiotic on mitochondria directly affects the rate of superoxide production, increasing rate of generation of $\mathrm{O}_{2} \bullet-$ by blocking the electron transport which enhance the reduction level of carriers present on inhibition site or if the xenobiotic accepts the electron from respiratory carrier and transfer to oxygen molecule by redox cycling, which also result in elevated level of $\mathrm{O}_{2} \bullet-[18]$. Thus, the clinical pathogenesis, apoptosis and aging is dependent to cellular respiration by regulation of ROS and RNS by mitochondria membrane potential.

\section{ER stress induces oxidative stress: Ca2+ connection}

The ER serves as a site for synthesis and proper folding of proteins through cascade of reactions that depends on the redox status within the lumen of ER [26]. In the lumen of ER, protein disulfide isomerase (PDI) enzymes catalyzes the disulfide bond formation in nascent proteins and accounts for balancing redox status of the environment [27]. Notably, the oxidizing environment of ER lumen contains high ratio of oxidized to reduced glutathione (GSSG/ GSH) for the synthesis of disulfide bonds during protein folding [3]. Concomitantly, cellular redox status reflects the balance between oxidized to reduced glutathione (GSSG/ GSH) and protein disulfide bond synthesis [28]. The consequences of ER stressinduced oxidative stress has detrimental effect on the stored $\mathrm{Ca}^{2+}$ ions within the lumen of ER [29]. The import and export of $\mathrm{Ca}^{2+}$ to the ER has been regulated by sarcoendoplasmic reticulum $\mathrm{Ca}^{2+}$ transport ATPase (SERCA) members and inositol 1,4,5-trisphosphate (IP ) $_{3}$ receptor $\left(\mathrm{IP}_{3} \mathrm{R}\right)$ members respectively [29]. An earlier report states that, the reduced GSH induced oxidative stress triggers release of $\mathrm{Ca}^{2+}$ from ER through inositol trisphosphate receptor $\left(\mathrm{IP}_{3} \mathrm{R}\right)[30]$.

In fact, excessive release ER stored in between of $\mathrm{Ca}_{2}{ }^{+}$to cytosol amplify the stress to mitochondria that leads to production of ROS through multiple mechanisms (Figure 1). Indeed, a fraction of the ER interacts with mitochondria known as mitochondria-associated ER membranes (MAMs) serves as a hotspots for the cross-talk between theses organelles [31]. It has been reported that, $5 \%$ to $20 \%$ fraction of mitochondria physically located to the ER serves as an exchange site of $\mathrm{Ca}^{2+}$ between ER to mitochondria through multiple channels [32]. In addition, the leaked $\mathrm{Ca}^{2+}$ from the ER via inositol triphosphate receptors $\left(\mathrm{IP}_{3} \mathrm{R}\right)$ accumulates and rapidly internalized within $\mathrm{t}$ e $\mathrm{h}$ mitochondria to generat excessive ROS, leading to alteration in mitochondrial membrane potential and mitochondrial damage $[33,34]$. Accumulation of $\mathrm{Ca}^{2+}$ within the mitochondria produces superoxide and creates oxidative stress [35]. In addition, calcium overload to mitochondria triggers 
mitochondrial dependent apoptotic instigation and lead to cell death [36]. In a feedback manner the mitochondrial $\mathrm{Na}^{+} / \mathrm{Ca}^{2+}$ exchanger (mNCX) releases some $\mathrm{Ca}^{2+}$ that taken up by the sarco- endoplasmic reticulum $\mathrm{Ca}^{2+}$-ATPase (SERCA) channel resides to ER [37-39].

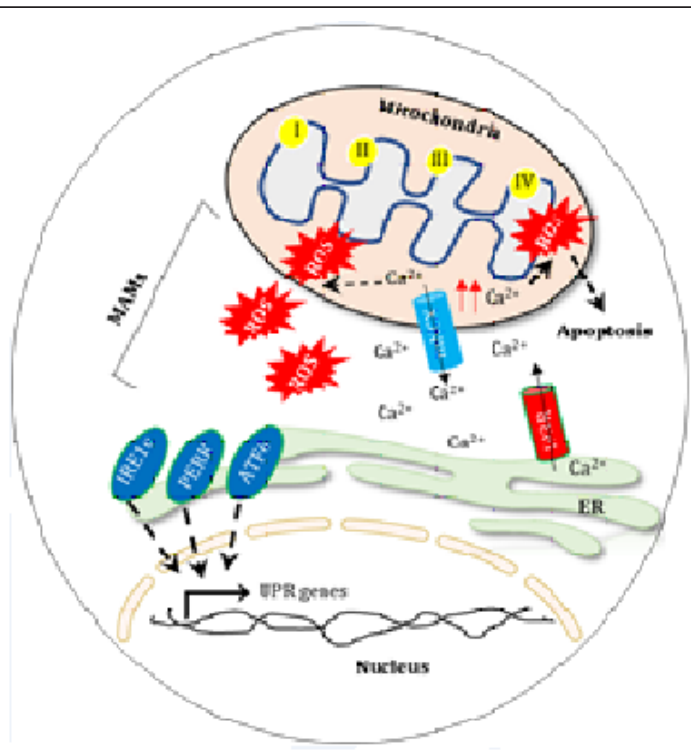

Figure 1: Oxidative regulation in Mitochondria and ER. Interface of mitochondria and ER (MAMs) shares an important site for the exchange of biomolecules and perturbation in balance leads to apoptotic cell death.

Moreover, the $\mathrm{Ca}^{2+}$ regulates opening if IP3R in a biphasic manner; at low concentration $\mathrm{Ca}^{2+}$ stimulates IP3R, whereas at high level $\mathrm{Ca}^{2+}$ inhibits $\mathrm{ER} \mathrm{Ca}^{2+}$ release [40]. Interestingly, the mitochondrial associated protein such as B-cell lymphoma 2 (Bcl-2), Bcl-2-associated X protein (Bax/Bak) and the Bcl-2interacting killer (BIK) may enhance $\mathrm{Ca}^{2+}$ transfer from ER to mitochondria leading to apoptosis via release of cytochrome $\mathrm{c}$ [12,41-43]. Indeed, levels of ROS can also tune $\mathrm{Ca}^{2+}$ release from ER; for instance, ROS at nanomolar concentrations enhances $\mathrm{Ca}^{2+}$ release through calmodulin-sensitized ryanodine receptor (RyR) whereas at micromolar concentrations ROS inhibits the function of calmodulin and restricts RyR activity [44]. Conversely, in a feedback mechanism the ROS originating from mitochondria triggers calcium release from ER to sensitize the calcium release channel at the ER membrane that reinforces ROS production in both organelles [45]. In addition, mitochondrial ROS can cross-influence ER functioning through dictating the cellular disulfide bond formation [46].

\section{ER-stress coping: UPR and Ca2+ signaling:}

In order to cope with exacerbated ER stress cells, activate unfolded protein response (UPR) through a signaling network consists of proteins IRE1 $\alpha$, PERK, and ATF6 $\alpha[47,48]$. The IRE1 $\alpha$, PERK, and ATF6 $\alpha$ are ER-spanning transmembrane proteins that interconnected through the ER chaperone BiP [47,49]. Indeed $\mathrm{BiP}$ has been reported in $\mathrm{Ca}^{2+}$ transport across the ER to the mitochondria through an ER membrane sigma-1 receptor (Sig-1R) [50]. Moreover, in response to ER stress IRE1 $\alpha$ autophosphorylates and activated splice X-box binding protein 1 (XBP1) mRNA that subsequently translocated into to the nucleus to control transcriptional machinery related to ER quality control and ERAD pathway [51]. Similar to IRE1 $\alpha$, PERK activation initiates phosphorylation of eukaryotic translation initiation factor $2 \alpha$ (eIF2 $\alpha$ ) to attenuates further translation machinery through activating Transcription Factor $4\left(\mathrm{ATF}_{4}\right)$ and restore ER stress [52]. In addition, the activation of ATF6 $\alpha$ generates the cleaved fragment of ATF $6 \alpha$ that translocate to the nucleus and induces transcriptional activation of UPR associated genes involved in IRE $\alpha$ and PERK signaling [53-55]. The ER Ca ${ }^{2+}$ imbalance can also activate PERK arm of UPR by interacting with calcineurin that act as a conserved $\mathrm{Ca}^{2+}$ activated phosphatase [56,57]. An earlier report shown that, ER stress subjected to PERK-knockout mouse fibroblasts leads to induction of ROS levels [28]. In contrast, UPR signaling network has been shown in regulation of ER-mitochondrial $\mathrm{Ca}^{2+}$ transfer through mitochondrial associated ubiquitin ligase Parkin [58,59]. In addition, the pro-apoptotic component of UPR such as CHOP may induce oxidative stress in response to ER stress. Notably, the CHOP target gene ERO1L contributes in disulfide bond formation during protein synthesis. In addition, ERO1 $\alpha$ regulates IP3R mediated $\mathrm{Ca}^{2+}$ leakage from the ER through interacting with kinase MKII and leading to the execution of oxidative stress induced apoptosis [6062].

\section{Conclusion}

The mitochondrial and ER associated membranes serves as regulatory sites for several biochemical dynamics relevant to human disease and remained elusive to further dissect. Failure to resolve cellular stress leads to results in pathological abnormalities and leading to cell death. For instance, mitochondria undergo 
continuous biochemical arrangement during mitochondrial respiration and generates several reactive oxygen species (ROS) molecules, however improper resolution of excessive ROS molecules leads to oxidative stress within cells. Similarly, ER lumen is the prime site for protein synthesis and folding, but immature protein folding within ER leads to transient ER stress that may results in cell death. In addition, mitochondria and ER serves as a storage site for calcium ions $\left(\mathrm{Ca}^{2+}\right)$ that play a key role in several pathologies. Notably, the transport channels such as IP3 and mNCX at the interface of ER and mitochondria respectively balance the excessive $\mathrm{Ca}^{2+}$ concentration in a feedback mechanism to minimize oxidative imbalance within cells. Importantly, the interface of mitochondria and endoplasmic reticulum (ER) are critical to cell functions including redox regulation, calcium signaling and lipid biosynthesis. Notably UPR signaling partially regulates the $\mathrm{Ca}^{2+}$ imbalance to the ER and reduce oxidative burden. Thus, a healthy tuning between mitochondria and ER determines the fate of oxidative stress resolution in the cells.

\section{Conflict of Interest}

Authors declares no conflict of interest.

\section{References}

1. Higa A, E Chevet (2012) Redox signaling loops in the unfolded protein response. Cell Signal 24(8): 1548-1555.

2. Perjes A, AM Kubin, A Konyi, S Szabados, A Cziraki, et al. (2012) Physiological regulation of cardiac contractility by endogenous reactive oxygen species. Acta Physiol (Oxf) 205(1): 26-40.

3. Chakravarthi S, NJ Bulleid (2004) Glutathione is required to regulate the formation of native disulfide bonds within proteins entering the secretory pathway. J Biol Chem 279(38): 39872-39879.

4. Malhotra JD, RJ Kaufman (2007) Endoplasmic reticulum stress and oxidative stress: a vicious cycle or a double-edged sword? Antioxid Redox Signal 9(12): 2277-2293.

5. Murphy MP (2009) How mitochondria produce reactive oxygen species. Biochem J 417(1): 1-13.

6. Scialo F, DJ Fernandez-Ayala, A Sanz (2017) Role of Mitochondrial Reverse Electron Transport in ROS Signaling: Potential Roles in Health and Disease. Front Physiol 8: 428.

7. Bourens M, F Fontanesi, IC Soto, J Liu, A Barrientos (2013) Redox and reactive oxygen species regulation of mitochondrial cytochrome $\mathrm{C}$ oxidase biogenesis. Antioxid Redox Signal 19(16): 1940-1952.

8. Holmstrom KM, T Finkel (2014) Cellular mechanisms and physiological consequences of redox-dependent signalling. Nat Rev Mol Cell Biol 15(6): 411-421.

9. Sevier CS, CA Kaiser (2008) Ero1 and redox homeostasis in the endoplasmic reticulum. Biochim Biophys Acta 1783(4): 549-556.

10. Harding HP, Y Zhang, H Zeng, I Novoa, PD Lu, et al. (2003) An integrated stress response regulates amino acid metabolism and resistance to oxidative stress. Mol Cell 11(3): 619- 633.

11. Tu BP, JS Weissman (2002) The FAD- and O(2)-dependent reaction cycle of Ero1-mediated oxidative protein folding in the endoplasmic reticulum. Mol Cell 10(5): 983-994.

12. Tabas I, D Ron (2011) Integrating the mechanisms of apoptosis induced by endoplasmic reticulum stress. Nat Cell Biol 13(3): 184-190.
13. Ranjan K, C Pathak (2016) Expression of FADD and cFLIPL balances mitochondrial integrity and redox signaling to substantiate apoptotic cell death. Mol Cell Biochem 422(1-2): 135-150.

14. Sugioka K, T Naruko, T Hozumi, M Nakagawa, C Kitabayashi, et al. (2010) Elevated levels of neopterin are associated with carotid plaques with complex morphology in patients with stable angina pectoris. Atherosclerosis 208(2): 524-530.

15. Becker LB, TL vanden Hoek, ZH Shao, CQ Li, PT Schumacker (1999) Generation of superoxide in cardiomyocytes during ischemia before reperfusion. Am J Physiol 277(6): H2240- H2246.

16. Sugioka K, M Nakano, I Naito, S Tero Kubota, Y Ikegami (1988) Properties of a coenzyme, pyrroloquinoline quinone: generation of an active oxygen species during a reduction-oxidation cycle in the presence of NAD(P)H and 02. Biochim Biophys Acta 964(2): 175-182.

17. Liu Y, G Fiskum, D Schubert (2002) Generation of reactive oxygen species by the mitochondrial electron transport chain. J Neurochem 80(5): 780787.

18. Turrens JF (2003) Mitochondrial formation of reactive oxygen species. J Physiol 552(Pt 2): 335-344.

19. Barja G (1999) Mitochondrial oxygen radical generation and leak: sites of production in states 4 and 3, organ specificity, and relation to aging and longevity. J Bioenerg Biomembr 31(4): 347-366.

20. Babior BM, JD Lambeth, W Nauseef (2002) The neutrophil NADPH oxidase. Arch Biochem Biophys 397(2): 342-344.

21. Chen Q EJ Vazquez, S Moghaddas, CL Hoppel, EJ Lesnefsky (2003) Production of reactive oxygen species by mitochondria: central role of complex III. J Biol Chem 278(38): 36027- 36031.

22. Kevin LG, AK Camara, ML Riess, E Novalija, DF Stowe (2003) Ischemic preconditioning alters real-time measure of 02 radicals in intact hearts with ischemia and reperfusion. Am J Physiol Heart Circ Physiol 284(2): H566-H574.

23. St-Pierre J, JA Buckingham, SJ Roebuck, MD Brand (2002) Topology of superoxide production from different sites in the mitochondrial electron transport chain. J Biol Chem 277(47): 44784-44790.

24. Amar SK, S Goyal, D Dubey, AK Srivastav, D Chopra, et al. (2015) Benzophenone 1 induced photogenotoxicity and apoptosis via release of cytochrome $\mathrm{c}$ and Smac/DIABLO at environmental UV radiation. Toxicol Lett 239(3): 182-193.

25. Goyal S, SK Amar, AK Srivastav, D Chopra, MK Pal, et al. (2016) ROS mediated crosstalk between endoplasmic reticulum and mitochondria by Phloxine $\mathrm{B}$ under environmental UV irradiation. J Photochem Photobiol B 161: 284-294.

26. Hampton RY (2002) ER-associated degradation in protein quality control and cellular regulation. Curr Opin Cell Biol 14(4): 476-482.

27. Zeeshan HM, GH Lee, HR Kim, HJ Chae (2016) Endoplasmic Reticulum Stress and Associated ROS. Int J Mol Sci 17(3): 327.

28. Haynes CM, EA Titus, AA Cooper (2004) Degradation of misfolded proteins prevents ER- derived oxidative stress and cell death. Mol Cell 15(5): 767-776

29. Berridge MJ, MD Bootman, HL Roderick (2003) Calcium signalling: dynamics, homeostasis and remodelling. Nat Rev Mol Cell Biol 4(7): 517-529.

30. Miyakawa T, A Mizushima, K Hirose, T Yamazawa, I Bezprozvanny, et al. (2001) $\mathrm{Ca}(2+)$-sensor region of IP(3) receptor controls intracellular $\mathrm{Ca}(2+)$ signaling. EMBO J 20(7): 1674-1680.

31. Annunziata I, R Sano, A d'Azzo (2018) Mitochondria-associated ER membranes (MAMs) and lysosomal storage diseases. Cell Death Dis $9(3): 328$.

32. Inoue T, H Maekawa, R Inagi (2019) Organelle crosstalk in the kidney. Kidney Int 95(6): 1318-1325. 
33. Bravo R, JM Vicencio, V Parra, R Troncoso, JP Munoz, et al. (2011) Increased ER- mitochondrial coupling promotes mitochondrial respiration and bioenergetics during early phases of ER stress. J Cell Sci 124(Pt 13): 2143-2152.

34. Diwan A, SJ Matkovich, Q Yuan, W Zhao, A Yatani, et al. (2009) Endoplasmic reticulum-mitochondria crosstalk in NIX-mediated murine cell death. J Clin Invest 119(1): 203-212.

35. Brookes PS, Y Yoon, JL Robotham, MW Anders, SS Sheu (2004) Calcium, ATP, and ROS: a mitochondrial love-hate triangle. Am J Physiol Cell Physiol 287(4): C817-C833.

36. Csordas G, G Hajnoczky (2009) SR/ER-mitochondrial local communication: calcium and ROS. Biochim Biophys Acta 1787(11): 1352-1362.

37. Berridge MJ (2009) Inositol trisphosphate and calcium signalling mechanisms. Biochim Biophys Acta 1793(6): 933-940.

38. Palty R, WF Silverman, M Hershfinkel, T Caporale, SL Sensi, et al. (2010) NCLX is an essential component of mitochondrial $\mathrm{Na}+/ \mathrm{Ca} 2+$ exchange. Proc Natl Acad Sci U S A 107(1): 436-441.

39. Luongo TS, JP Lambert, P Gross, M Nwokedi, AA Lombardi, et al. (2017) The mitochondrial $\mathrm{Na}(+) / \mathrm{Ca}(2+)$ exchanger is essential for $\mathrm{Ca}(2+)$ homeostasis and viability. Nature 545(7652): 93-97.

40. Iino M, M Endo (1992) Calcium-dependent immediate feedback control of inositol 1,4,5- triphosphate-induced Ca2 + release. Nature 360(6399): 76-78.

41. Ranjan K, C Pathak (2016) FADD regulates NF-kappaB activation and promotes ubiquitination of cFLIPL to induce apoptosis. Sci Rep 6: 22787

42. Ranjan K, A Sharma, A Surolia, C Pathak (2014) Regulation of HA14-1 mediated oxidative stress, toxic response, and autophagy by curcumin to enhance apoptotic activity in human embryonic kidney cells. Biofactors 40(1): 157-169.

43. Ranjan K, C Pathak (2016) Expression of cFLIPL Determines the Basal Interaction of Bcl-2 With Beclin-1 and Regulates p53 Dependent Ubiquitination of Beclin-1 During Autophagic Stress. J Cell Biochem 117(8): 1757-1768.

44. Okabe E, Y Tsujimoto, Y Kobayashi (2000) Calmodulin and cyclic ADPribose interaction in $\mathrm{Ca} 2+$ signaling related to cardiac sarcoplasmic reticulum: superoxide anion radical-triggered $\mathrm{Ca} 2+$ release. Antioxid Redox Signal 2(1): 47-54.

45. Bhandary B, A Marahatta, HR Kim, HJ Chae (2012) An involvement of oxidative stress in endoplasmic reticulum stress and its associated diseases. Int J Mol Sci 14(1): 434-456.

46. Ishii T, O Sunami, H Nakajima, H Nishio, T Takeuchi, et al. (1999) Critical role of sulfenic acid formation of thiols in the inactivation of glyceraldehyde-3-phosphate dehydrogenase by nitric oxide. Biochem Pharmacol 58(1): 133-143.

47. Cubillos Ruiz JR, SE Bettigole, LH Glimcher (2017) Tumorigenic and Immunosuppressive Effects of Endoplasmic Reticulum Stress in Cancer. Cell 168(4): 692-706.

48. Gardner BM, D Pincus, K Gotthardt, CM Gallagher, P Walter (2013) Endoplasmic reticulum stress sensing in the unfolded protein response. Cold Spring Harb Perspect Biol 5(3): a013169.
49. Chang TK, DA Lawrence, M Lu, J Tan, JM Harnoss, et al. (2018) Coordination between Two Branches of the Unfolded Protein Response Determines Apoptotic Cell Fate. Mol Cell 71(4): 629-636.

50. Dudek J, J Benedix S Cappel, M Greiner, C Jalal, et al. (2009) Functions and pathologies of BiP and its interaction partners. Cell Mol Life Sci 66(9): 1556-1569.

51. Calfon M, H Zeng, F Urano, JH Till, SR Hubbard, et al. (2002) IRE1 couples endoplasmic reticulum load to secretory capacity by processing the XBP-1 mRNA. Nature 415(6867): 92-96.

52. Rowlands AG, R Panniers, EC Henshaw (1988) The catalytic mechanism of guanine nucleotide exchange factor action and competitive inhibition by phosphorylated eukaryotic initiation factor 2. J Biol Chem 263(12): 5526-5533.

53. Ye J, RB Rawson, R Komuro, X Chen, UP Dave, et al. (2000) ER stress induces cleavage of membrane-bound ATF6 by the same proteases that process SREBPs. Mol Cell 6(5): 1355-1364.

54. Yamamoto K, T Sato, T Matsui, M Sato, T Okada, et al. (2007) Transcriptional induction of mammalian ER quality control proteins is mediated by single or combined action of ATF6alpha and XBP1. Dev Cell 13(3): 365-376.

55. Fornace AJ, I Alamo, MC Hollander (1988) DNA damage-inducible transcripts in mammalian cells. Proc Natl Acad Sci U S A 85(23): 88008804.

56. Wang R, BC McGrath, RF Kopp, MW Roe, X Tang, et al. (2013) Insulin secretion and $\mathrm{Ca} 2+$ dynamics in beta-cells are regulated by PERK (EIF2AK3) in concert with calcineurin. J Biol Chem 288(47): 3382433836.

57. Bollo M, RM Paredes, D Holstein, N Zheleznova, P Camacho, et al. (2010) Calcineurin interacts with PERK and dephosphorylates calnexin to relieve ER stress in mammals and frogs. PLoS One 5(8): e11925.

58. Bouman L, A Schlierf, AK Lutz, J Shan, A Deinlein, et al. (2011) Parkin is transcriptionally regulated by ATF4: evidence for an interconnection between mitochondrial stress and ER stress. Cell Death Differ 18(5): 769-782.

59. Cali T, D Ottolini, A Negro, M Brini (2013) Enhanced parkin levels favor ER-mitochondria crosstalk and guarantee $\mathrm{Ca}(2+)$ transfer to sustain cell bioenergetics. Biochim Biophys Acta 1832(4): 495-508.

60. Han J, SH Back, J Hur, YH Lin, R Gildersleeve, et al. (2013) ER-stressinduced transcriptional regulation increases protein synthesis leading to cell death. Nat Cell Biol 15(5): 481-490.

61. Li G, M Mongillo, KT Chin, H Harding, D Ron, et al. (2009) Role of ER01alpha-mediated stimulation of inositol 1,4,5-triphosphate receptor activity in endoplasmic reticulum stress-induced apoptosis. J Cell Biol 186(6): 783-792.

62. Marciniak SJ, CY Yun, S Oyadomari, I Novoa, Y Zhang, et al. (2004) CHOP induces death by promoting protein synthesis and oxidation in the stressed endoplasmic reticulum. Genes Dev 18(24): 3066-3077. 